\title{
Scaled momentum distributions and energy dependence of charged multiplicities
}

\author{
Teresa Tymieniecka ${ }^{1}$ \\ on behalf of the ZEUS Collaboration \\ 1- University of Warsaw - Faculty Of Physics \\ ul.Hoża 69, 00681 Warszawa - Poland
}

\begin{abstract}
The charged multiplicity distributions, the mean charged multiplicity and the scaled momentum distributions have been investigated in semi-inclusive neutral current deep inelastic $e p$ scattering with the ZEUS detector at HERA. The measurements are performed in the current region of the Breit frame, as well as in the current fragmentation region of the hadronic centre-of-mass frame. The KNO-scaling properties of the data are investigated using different energy scales. The data are compared with similar measurements obtained in $e^{+} e^{-}$annihilation and with previous ep measurements as well as with leading-logarithm parton-shower Monte Carlo predictions.
\end{abstract}

\section{Introduction}

The study of parton fragmentation and hadronisation processes provides valuable insight into the nonperturbative regime of Quantum Chromodynamics (QCD). These processes are studied at the HERA energies using the semi-inclusive charged particles produced in neutral currant deep inelastic scattering. The measurements of particles are performed in the current region of the Breit frame and of the hadronic centre-of-mass (HCM) frame. The current region of the HCM frame is investigated in bins of the total hadronic energy $W$. In the Breit frame the exchanged boson virtuality, $Q$ is related to the momentum of the scattered parton, $Q / 2$. Its hadronisation features are compared with similar observables measured in one hemisphere of hadronic final states in $e^{+} e^{-}$annihilation described by half of the centre-

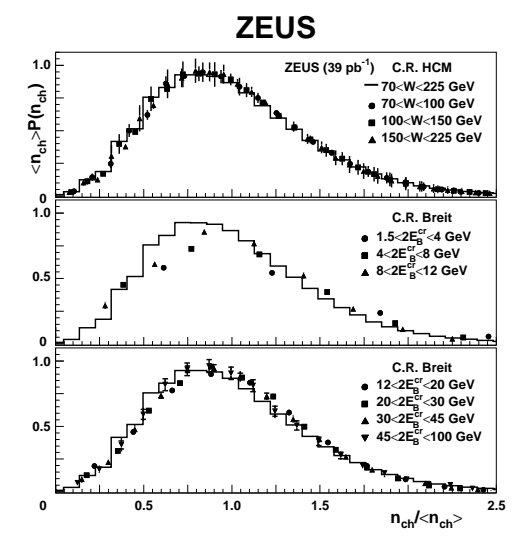

Figure 1: The multiplicity distributions plotted in KNO form. of-mass energy, $E^{*}$. An alternative energy scale to $Q$ is considered to be either the available energy in the Breit current region $E_{B}^{c r}$ or the invariant mass of the emitted particles $M_{\text {eff }}^{2}$. For both the sum runs over all charged and neutral particles of the corresponding hadronic system.

\section{Multiplicity of the charged particles}

The data were collected with the ZEUS detector during the 1996 and 1997 running periods, when HERA operated with protons of energy $E_{p}=820 \mathrm{GeV}$ and positrons of energy $E_{e}=27.5 \mathrm{GeV}$, and correspond to an integrated luminosity of $38.6 \pm 0.6 \mathrm{pb}^{-1}$. The analysis is described in some detail elsewhere[2].

DIS 2008 
Nearly forty years ago Koba-Nielsen-Olesen (KNO) [3] put forward the hypothesis that at very high energies $s$ the probability distribution $P_{n}(s)$ of producing $n$ particles in a certain collision process should exhibit the scaling relation $P_{n}(s)=\frac{1}{\langle n(s)\rangle} \psi\left(\frac{n}{\langle n(s)\rangle}\right)$ as $s \rightarrow \infty$ with $\langle n(s)\rangle$ being the average multiplicity of secondaries at collision energy $s$. As seen in Fig. 1 the rescaled multiplicity points $\left\langle n_{\mathrm{ch}}\right\rangle \cdot P\left(n_{c h}\right)$ measured at different energies form the unique scaling curve as a function of the scaled multiplicity $\frac{n_{c} h}{\left\langle n_{c} h\right\rangle}$. The KNO distributions measured in bins of $W$ in the current region of the HCM frame agree with the reference distribution represented by the average distribution (histogram) which is calculated using data for the entire $W$ region, $70<W<225 \mathrm{GeV}$. The reference KNO distribution is also compared to the measurements in the current region of the Breit frame. The points follow the KNO-scaling behaviour if the available energy $2 * E_{B}^{c r}$ is large enough (here $\left.2 * E_{B}^{c r}>12 \mathrm{GeV}\right)$. The scale $2 * E_{B}^{c r}$ characterizes the total centre-of-mass energy of the system of which only one hemisphere is measured.

\section{ZEUS}

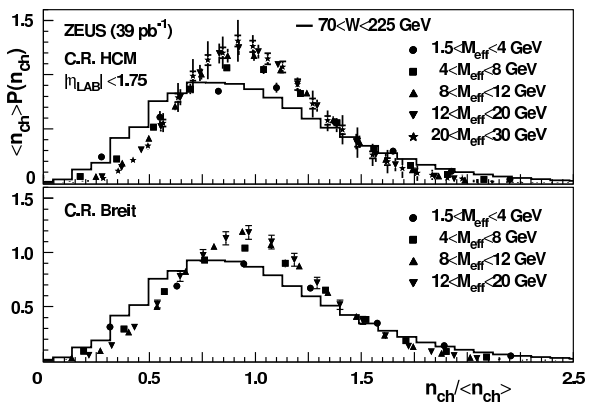

Figure 3: The multiplicity distributions plotted in KNO form are compared with the reference KNO distribution (histogram) and with measurements in bins of $M_{\text {eff }}^{2}$ in the current regions.

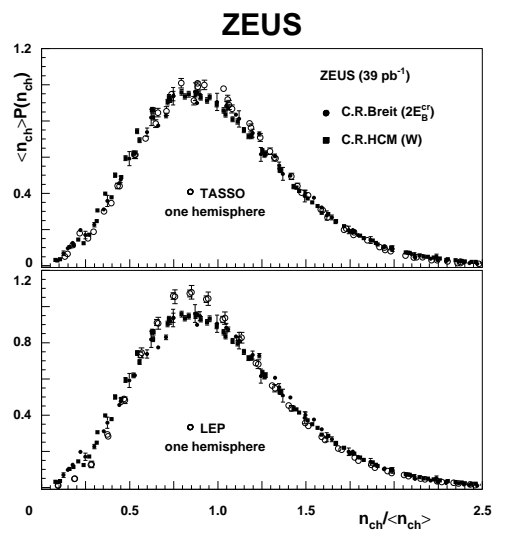

Figure 2: The multiplicity distributions as in Fig. 1 but for $2 *$ $E_{B}^{c r}>12 \mathrm{GeV}$. The $e p$ data are compared with $e^{+} e^{-}$measurements (open points). $M_{\text {eff }}^{2}$ for $M_{\text {eff }}^{2}>8 \mathrm{GeV}$ are compared in Fig. 4 with the $e^{+} e^{-}$measurements of TASSO and LEP for the whole event. The agreement is good. Finally, the mean charged multiplicities measured in the current regions of the Breit and HCM frames as a function of the respective available energies, $2 * E_{B}^{c r}$ and $W$ are presented in Fig. 5 . The DIS data are scaled by a factor 2 since they are measured in one hemisphere. The DIS fixed target data are also corrected for the $K^{0}$ and $\Lambda$ decays (a factor 1.08). All the measurements exhibit approximately the same dependence of the mean charged multiplicity on the respective energy scale except the data from the DIS fixed target experiments which deviate at energies above $15 \mathrm{GeV}$. More 
information on multiplicity characteristics can be found elsewhere [2].

\section{Scaled momentum distributions}

The scaled momentum spectra have been studied using an integrated luminosity of about $0.5 \mathrm{fb}^{-1}$ taken with the ZEUS detector at HERA [7]. The scaled particle momentum $x_{p}$ is defined as the moscaled by the maximum momentum $Q / 2$ of a quark in the current region. The evolution of scaled momentum distributions is shown as a function of the available energy defined in $e p$ scattering by the exchanged boson virtuality $Q^{2}$ (Fig. 6). Scaling violations are observed. The evolution is compared with the evolution of the similar distributions obtained from the $\mathrm{H} 1$ measurements [8] and from $e^{+} e^{-}$experiments $[9,10,11,12]$.

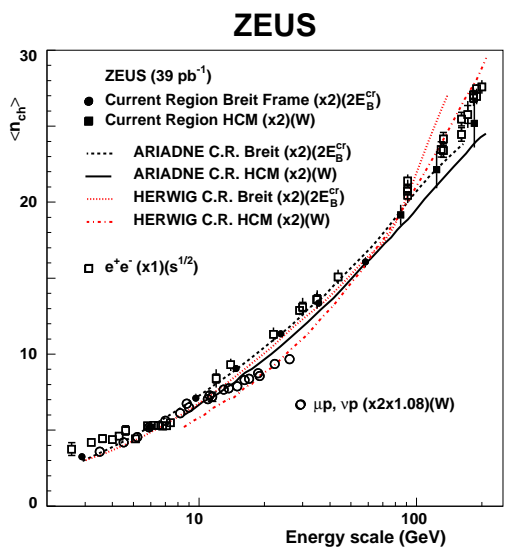

Figure 5: Mean charged multiplicity $\left\langle n_{\mathrm{ch}}\right\rangle$ multiplied by 2 plotted as a function of $2 * E_{B}^{c r}$ and $W$ respectively for the current regions of the Breit and HCM frames. The measurements from $e^{+} e^{-}$and the fixed target experiments (see text) are also plotted. frame. mentum of a particle measured in the Breit frame

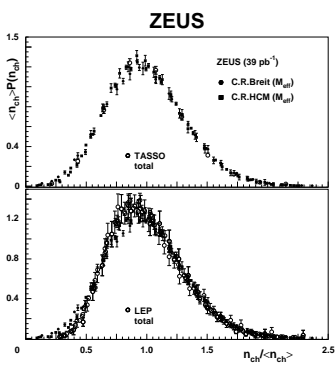

Figure 4: The ZEUS data as in Fig. 3 compared with the similar observables from $e^{+} e^{-}$annihilation measured for the whole event.

There is good agreement between different sets of $e p$ data. The $e p$ data show similar behaviour to the $e^{+} e^{-}$data providing a rough demonstration of fragmentation universality. However, a detailed comparison between $e^{+} e^{-}$and $e p$ shows some discrepancies. At high values of $Q^{2}$ approaching the $Z^{0}$ exchanged region and at $x_{p}<0.1$ there are less charged particles observed in the $e p$ data than in $e^{+} e^{-}$annihilation. Similar observations at $Q^{2}<100 \mathrm{GeV}^{2}$ in previous measurements have been understood in terms of higher order QCD processes which deplete the current region in the Breit frame and populate the region between the origin of the Breit frame and the origin of the HCM

\section{$4 \quad$ Summary and conclusions}

The charged multiplicity distributions and the mean charged multiplicity have been investigated in inclusive neutral current deep inelastic ep scattering in the kinematic range $Q^{2}>25 \mathrm{GeV}^{2}$ and $70<W<225 \mathrm{GeV}$ in terms of different energy scales. The scale $2 * E_{B}^{c r}$, was used in the current region of the Breit frame. In the current region of the HCM frame, $W$ was used and the invariant mass, $M_{\text {eff }}^{2}$, was used in both frames.

In terms of KNO scaling, the charged multiplicities in the current regions of the Breit and HCM frames exhibit the same behaviour as those in one hemisphere of $e^{+} e^{-}$collisions when 
$2 * E_{B}^{c r}$ or $W$ are considered. When the energy scale $M_{\text {eff }}^{2}$ is used, the charged multiplicities exhibit the same KNO-scaling behaviour as those for the whole $e^{+} e^{-}$event if $M_{\text {eff }}^{2}$ is sufficiently large.

Comparison of the scaled momentum distributions of charged particles produced in $e p$ scattering with the same observables measured in $e^{+} e^{-}$ annihilation support broadly the concept of quark fragmentation universality. Some depletion of the scaled momentum distributions at low $Q^{2}$ can be attributed to higher order QCD processes occurring in $e p$ but not in $e^{+} e^{-}$. At high $Q^{2}$ approaching the $Z^{0}$ exchanged region a significant depletion is also observed.

\section{Acknowledgments}

The author would like to think the Workshop organizers for the excellent organization, hospitality and some financial support.

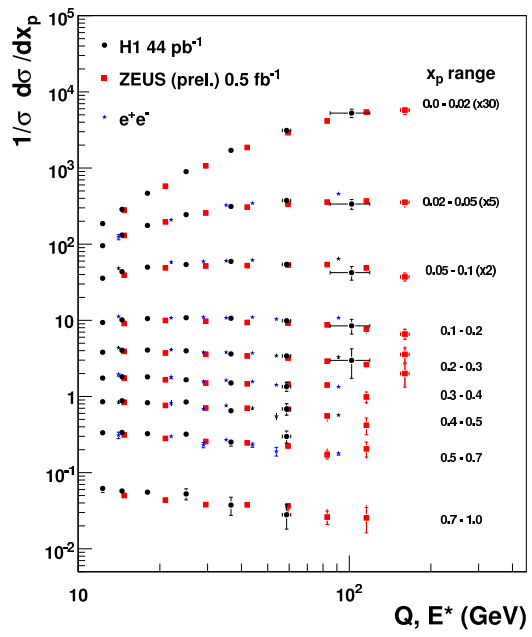

Figure 6: The number of charged particles per interaction given in form of $1 / \sigma d \sigma / d x_{p}$ in the current region of the Breit frame as a function of the virtuality $Q$ for the ZEUS and H1 data in $x_{p}$ bins compared with $e^{+} e^{-}$data plotted as a function of $E^{*}$.

[1] Slides: http://indico. cern.ch/contributionDisplay py? contribId=203\&sessionId=13\&conf Id=24657

[2] ZEUS Collaboration, S. Chekanov et al., JHEP, DESY08-036, 2008 and references there.

[3] Z. Koba, H.B. Nielsen and P. Olesen, Nucl.Phys. 40 317 (1972).

[4] TASSO Collaboration, W. Braunschweig et al., Z. Phys. C45 193 (1989).

[5] OPAL Collaboration, P.D. Acton et al.,, Z. Phys. C35 539 (1991).

[6] DELPHI Collaboration, P. Abreu et al., Z. Phys. C50 185 (1991)

[7] http://www-zeus.desy.de/public_results/searchdbpublic.php(ZEUS-prel-07-006)

[8] H1 Collaboration, F.D. Aaron et al., Phys. Lett. B654 148 (2007)

[9] TASSO Collaboration, W. Braunschweig et al., Z. Phys. C47 187 (1990).

[10] MARK II Collaboration, A. Petersen et al., Phys. Rev. D37 1 (1988).

[11] DELPHI Collaboration, P. Abreu et al., Phys. Lett. B311 408 (1993).

[12] AMY Collaboration, Y.K. Li et al., Phys. Rev. D41 2675 (1990). 